\title{
Nexus Among Energy Consumption Structure, Energy Intensity, Population Density, Urbanization And Carbon Intensity: A Heterogeneous Panel Evidence Considering Differences In Electrification Rates
}

\section{Jingqi Sun}

North China Electric Power University - Beijing Campus: North China Electric Power University

Xiaohui Guo (D951910616@qq.com )

North China Electric Power University - Beijing Campus: North China Electric Power University

\section{Yuan Wang}

North China Electric Power University - Beijing Campus: North China Electric Power University

Jing Shi

Kunming Bureau of China Southern Power Grid EHV Transmission Company,Kunming 650000,China

Yiquan Zhou

North China Electric Power University - Beijing Campus: North China Electric Power University

Boyang Shen

University of Cambridge Central Science Library: University of Cambridge

\section{Research Article}

Keywords: carbon intensity, electrification rate, heterogeneous panel analysis, convergence analysis

Posted Date: July 12th, 2021

DOl: https://doi.org/10.21203/rs.3.rs-581133/v1

License: (c) (i) This work is licensed under a Creative Commons Attribution 4.0 International License. Read Full License

Version of Record: A version of this preprint was published at Environmental Science and Pollution Research on October 29th, 2021. See the published version at https://doi.org/10.1007/s11356-02117165-3. 
1 Nexus among energy consumption structure,

2 energy intensity, population density, urbanization

3 and carbon intensity: a heterogeneous panel

4 evidence considering differences in electrification

5 rates

$6 \quad$ Jingqi Sun 1, Xiaohui Guo 1*, Yuan Wang 1, Jing Shi 2, Yiquan Zhou 1 and Boyang Shen 3

71 School of Economics and Management, North China Electric Power University, Beijing 102206, China;

8 sjq@ncepu.edu.cn(J.S.); 951910616@qq.com(X.G.); 1797763061@qq.com(Y.W.); 478747109@qq.com(Y.Z.)

92 Kunming Bureau Of China Southern Power Grid EHV Transmission Company, Kunming 650000, China;

10 shijing0421@hotmail.com(J.S.)

113 Electrical Engineering Division, Department of Engineering, University of Cambridge, Cambridge CB3 0FA,

12 UK; bs506@cam.ac.uk(B.S.)

13 Abstract:

14 In this paper, the clustering method is used to divide the 30 provinces of the country into

15 high, medium and low electrification rates according to the electrification rate from 2000 to

16 2017. The heterogeneous panel technology is used to analyze the relationship of energy

17 consumption structure, energy intensity, population density, urbanization rate and carbon

18 intensity. According to Cross-sectional dependence(CD) test and cross-section Im-Pesaran-

* the corresponding author 
Shin (CIPS) test results, the data of each panel are not in the form of same order single integer, so $\alpha$ convergence analysis, $\beta$ absolute convergence, and $\beta$ conditional convergence analysis are required. The results show that the carbon intensity of the four panels shows an $\alpha$ convergence; the $\beta$ absolute convergence shows there is a "catch-up effect"; $\beta$ conditional convergence indicates that the carbon intensity approaches their respective steady state levels; there is a long-term equilibrium relationship of energy consumption structure, energy intensity, population density and carbon intensity in all panels, but the urbanization rate has a significant impact on carbon intensity only in areas with high electrification rates. Finally, based on the results of empirical research, policy recommendations for reducing the carbon intensity in different regions are proposed.

Keywords: carbon intensity; electrification rate; heterogeneous panel analysis; convergence analysis

\section{Introduction} labor is clearer, and the growth rate has changed from high-speed growth to medium-high growth. China's economic development model is changing from extensive growth that focuses on scale and speed to intensive growth that focuses on quality and efficiency. The economic structure is changing from incremental expansion to stock adjustment, and the economic development momentum is changing from the traditional growth point to a new growth point. resources. 
At the same time, in China's economic development and urbanization process, a large

41 amount of energy consumption plays a key role, leading to increasing carbon emissions and increasing negative environmental impacts. Free Trade Area, Americans worried that free trade would worsen the Mexican environment and affect the domestic environment of the United States. They conducted the first empirical study on the relationship between environmental quality and per capita income, and put forward the EKC hypothesis(Grossman et al.1991). Soumyananda D explained the Environmental Kuznets Curve (EKC) hypothesis postulates an inverted-U-shaped relationship between different pollutants and per capita income, i.e., environmental pressure increases up to a certain level as income goes up; after that, it decreases(Soumyananda D.2004).

51 In early research, various environmental pollution indicators, cross-sectional data, and panel

52 data to explore environmental issues related to economic development are used through dual

53 empirical models to verify the EKC assumption, but no consistent conclusion was achieved. In

54 China, output and carbon dioxide emissions are not just inverse relations(Lu H.2000).

55 Azomahou and Van Phu used non-parametric research methods, showing that the relationship 56 between carbon dioxide emissions and GDP is more complicated than the EKC 57 curve(Azomahou et al.2001). Galeotti and Lanza used panel data to calculate the relationship 58 between $\mathrm{CO}_{2}$ emissions and GDP, and predicted emissions, believed that the empirical 59 relationship between two variables is in a non-linear form, rather than the linear or logarithmic 60 functions commonly considered(Galeotti M et al.1999). 

researchers gradually added various factors to explore the relationship between GDP and $\mathrm{CO}_{2}$ emissions from a variety of perspectives. Ma XJ and aims to quantify the relation between real GDP, $\mathrm{CO}_{2}$ emissions, renewable and nonrenewable energy consumption, tourism development and labor force for France and Germany. Results reveal an inverted U-shape relation between $\mathrm{CO} 2$ emissions and real GDP in long run confirming the validity of environmental Kuznets curve for the group of France and Germany(Ma XJ et al.2021). Anser has identified the causality between GDP growth and carbon emission and found bidirectional causality between economic growth and energy use(Anser Muhammad Khalid et al.2021).

71 In addition, urbanization is an important factor in regard to the relationship between GDP and $\mathrm{CO}_{2}$ emissions. For example, Wang $\mathrm{Z}$ investigates the dynamic interdependence between

$73 \mathrm{CO} 2$ emissions, real gross domestic product (GDP), renewable and non-renewable energy

74 generation, urbanization, and export quality for both the top ten renewable energy and top ten 75 economic complexity index (ECI) countries (Wang $\mathrm{Z}$ et al.2021). Faisal investigates the 76 association among carbon dioxide emissions, electricity consumption, capital, financial 77 deepening and urbanization, the long-run effects identified the evidence of an inverted U78 shaped association among carbon dioxide and urbanisation. This suggests that rapid 79 urbanisation increases the levels of pollution in the initial stages of development(Faisal et 80 al.2021). De uses parametric and semiparametric panel data analysis methodologies to test the 81 hypothesis of the environmental Kuznets curve, in 186 countries in the period 1960-2019. The 
83 and economic growth (GDP) and urbanization (\% population) in the parametric models(De et 84 al.2021).

85 With the rapid economic development, carbon dioxide emissions have increased significantly, and environmental problems have become more and more serious. However, the total indicators have limitations in studying economic and environmental issues. Compared with the total index, the total index reflects the relative number of comprehensive changes in multiple items or variables, the carbon emission intensity index has more complex composition and influencing factors. Its form depends on the carbon emission coefficient of carbon-based energy, the composition of carbon-based energy, the proportion and energy intensity of carbon-based energy in total energy consumption, so that we can grasp timely and effectively the frontier areas and development trends of carbon emission intensity research. Many foreign scholars have begun to conduct research on carbon emission intensity. This research began in 1997. Reborts JT and Grimes PE compiled relevant data on the economic development and carbon emissions of several developed countries from 1965 to 1992 to verify carbon emissions. The correlation between intensity and national economic output, and the conclusion that both passed the "EKC curve" test, which opened the prelude to carbon emission intensity research(Roberts J T et al.1997).

100 The environmental pollution in China is affected by technological progress, economic 101 growth, changes in industrial structure and the process of urbanization, as well as economic 102 cycles and fluctuations, which is uncertain and hard to predict. In the past, studies were limited 
103 in the relationship between economic intensity and carbon intensity, but economic volatility in

104 different cycles was relatively large. Carbon intensity was not easy to predict, and with

105 economic development, the factors affecting carbon intensity became broader. Therefore, many

106 domestic scholars have analyzed the influencing factors of carbon emission intensity.

107 In terms of influencing factors, Zhu and Zhang used the Logarithmic Mean Divisia Index

108 (LMDI) model to analyze the influencing factors of energy intensity (EI) in Shanghai from 1995

109 to 2008 (Zhu L et al.2011). The results show that the main influencing factor is the energy

110 intensity of the industrial sector, followed by the energy structure adjustment and industrial

111 structure adjustment. Gao of Lanzhou University analyzed the factors influencing the changes

112 of carbon emissions in Sichuan Province from 2000 to 2011 through the LMDI model(Gao L et

113 al.2014). Wang found that technological progress is not the main factor. By analyzing the spatial

114 spillover effects and driving factors of carbon emission intensity in 283 cities across the country

115 from 1992 to 2013, he found that the main influencing factors are also different in regions with

116 different levels of carbon intensity(Wang $\mathrm{S}$ et al.2019).

117 Dong and $\mathrm{Xu}$ based on the multiplication and addition factor decomposition of the Mean

118 Dirichlet Index (LMDI) to examine the impact of the four driving factors of energy intensity,

119 internal energy structure, economic structure and external energy structure on the carbon

120 intensity fluctuations in the production sector. The study found that energy intensity and

121 internal energy structure are negative driving factors, while economic structure and external

122 energy structure are positive driving factors(Dong M et al.2019). Based on perfect indicators

123 and dynamic spatial panel model, Zhang F et al established a comprehensive framework to 

logarithmic average weight Divisia decomposition method to construct a factor decomposition model of Beijing, Tianjin and Hebei's overall, regional, and industrial carbon intensity, and conducted an empirical analysis(Wang S et al.2017). Based on the statistical data of 30 provinces,

136 municipalities and autonomous regions in China from 2004 to 2016, Feng, Zhang and Wang

137 used Kernel density estimation method and LMDI factor decomposition method to study and 138 analyze the dynamic evolution characteristics and main influencing factors of China's direct

139 living energy consumption carbon intensity(Feng S et al.2018). On the basis of estimating the 140 carbon intensity of each province, Liu Xianzhao, Gao Changchun and others used exploratory 141 spatial data analysis (ESDA), spatiotemporal transition measurement methods and geographic 142 weighted regression (GWR) models to analyze China's provinces from 1995 to 2017. The spatial 143 dependence pattern of energy consumption, carbon intensity and the spatial heterogeneity of 144 its driving factors(Liu X et al.2018). 

and consumption, support the coordinated economic and social development, and promote the ecological environment. Therefore, the electrification rate is closely related to electricity, economy and environment. However, the existing research often ignores the factor of electrification rate. In addition, due to the significant heterogeneity of China's geographic map

151 (Fei et al.2011, Zhang C et al.2012), this leads to deviations in traditional panel analysis 152 techniques based on homogeneous panel data. Therefore, the heterogeneous panel analysis 153 method should be used.

154 In summary, carbon emission from energy consumption is an inevitable by-product of 155 economic development. Generally speaking, areas with large populations and high density 156 have more carbon emission, which are also closely related to economic growth, so population 157 density is related to carbon intensity. Moreover, energy intensity reflects the economy's 158 dependence on energy and is also related to carbon intensity to a certain extent. Furthermore, 159 with the development of urbanization, urbanization rate has also become a potential factor 160 affecting carbon emissions. Therefore, based on the regional diversity, this article mainly 161 studies the relationship between electricity, economy, energy and the environment, through 162 classifying 30 provinces by the electrification rate for energy-saving emission reduction policies.

163 The innovations of this article are as follows:

164 (1) At present, a new round of scientific and technological revolution has promoted the 165 development of electrification into a new historical stage. The development of energy and 
power is greener, the transmission and use of power is more intelligent, and the integration of

167 energy and power with economic society and people's lives is closer, the power-centric energy

168 transformation and upgrading is being accelerated. Therefore, the classification on 30

169 provinces in China based on the electrification rate is proposed to study the relationship

170 between China's electricity, economy, energy and environment.

171 (2) According to the electrification rate of each province, 30 provinces in China are divided

172 into three regions: high electrification rate, medium electrification rate and low electrification

173 rate. In addition, heterogeneous panel technology is used to study the relationship between

174 energy intensity, carbon intensity, energy consumption structure, urbanization rate and

175 population density in different regions, which helps to formulate corresponding carbon

176 emission reduction policies according to the characteristics of regional development.

177 (3) The heterogeneity between provincial panels in China is considered in this paper, so the

178 heterogeneous panel analysis techniques are employed, including the CD cross-section

179 correlation test and the second-generation unit root test CIPS test to ensure the validity of the

180 results. Because the variables are non-homogeneous and simple integers in different panels, $\alpha$

181 convergence analysis, $\beta$ absolute convergence analysis, and $\beta$ conditional convergence analysis

182 are performed for each variable in different panels.

183 The rest of the article is organized as follows: in Section 2, data resource, descriptive statistics

184 of variables, and the model adopted in this paper are presented. In Section 3, the major

185 econometric methodologies are proposed. In Section 4, the empirical findings and the 


\section{Proposed model, data and descriptive statistics}

\subsection{Proposed model and data} urbanization, energy factors and economic growth have significant effects on $\mathrm{CO}_{2}$ emissions. To further explore the interrelationships among energy intensity, urbanization rate, energy consumption structure, population density and carbon intensity, this paper proposes an expanded model where five representative variables are involved, shown as Eq.(1). dimension is year) . CI means the carbon intensity (ten thousand tons / 100 million RMB), i.e., the $\mathrm{CO}_{2}$ emission per unit of GDP. UR is the urbanization rate counted by proportion of urban population to the total population ECS reflects the structure of energy consumption, indicating that coal consumption accounts for the proportion of total energy consumption. $P D$ means the 201 population density (person / square kilometer). EI means the energy intensity, that is, energy 202 consumed per unit of GDP (10,000 tons of standard coal / 100 million RMB). The data of 203 province-level $\mathrm{CO}_{2}$ emissions are obtained from China Emission Accounts and Datasets, and 204 other data are collected from National Statistics Bureau. To reduce data fluctuations and 205 eliminate the heteroscedasticity that may exist in the panel, the variables $P D$ is converted in 
variables, their coefficient can stand for the elasticity directly. According to previous related researches [Zhu Z et al.2017, Lotfalipour M R et al.2010, Arouri M E H et al.2012], the logarithmic linear function is constructed as bellow:

$$
C I_{a t}=\beta_{1 a} U R_{a t}+\beta_{2 a} E C S_{a t}+\beta_{3 a} L P D_{a t}+\beta_{4 a} E I_{a t}+u_{a b}
$$

211 where $\beta_{1 a}, \beta_{2 a}, \beta_{3 a}$ and $\beta_{4 a}$ are elasticities of $C I$ with pertain to UR, ECS, LPD and EI per

212 province, respectively. $\mu_{a b}$ is the error with a mean of 0 and a variance of $\sigma^{2}$.

214 We use a balanced panel of 30 provinces in China, namely, Tianjin (TJ), Beijing (BJ), Shanghai 215 (SH), Inner Mongolia (IM), Shandong (SD), Chongqing (CQ), Hubei (HuB), Jiangsu (JS) , Jilin 216 (JL), Shaanxi (SX1), Liaoning (LN), Ningxia (NX), Hunan (HuN), Guangdong (GD), Hainan 217 (HaiN), Qinghai (QH), Hebei (HeB), Henan (HeN), Xinjiang (XJ), Zhejiang (ZJ), Heilongjiang 218 (HLJ), Jiangxi (JX), Sichuang (SC), Anhui (AH), Guangxi (GX), Shanxi(SX2), Guizhou(GZ), 219 Yunnan (YN) and Gansu (GS), Fujian (FJ), covering the period from 2000 to 2017.

220 As socialism with Chinese characteristics enters a new era, accurately grasp the level of 221 electrification development in various regions of our country, deeply analyze the development 222 potential of electrification, scientific planning, policy guidance, and key advancement, and 223 continuously improve the level of electrification in China. This is an important way to promote 224 high-quality economic development and the process of low-carbon energy and electricity. 


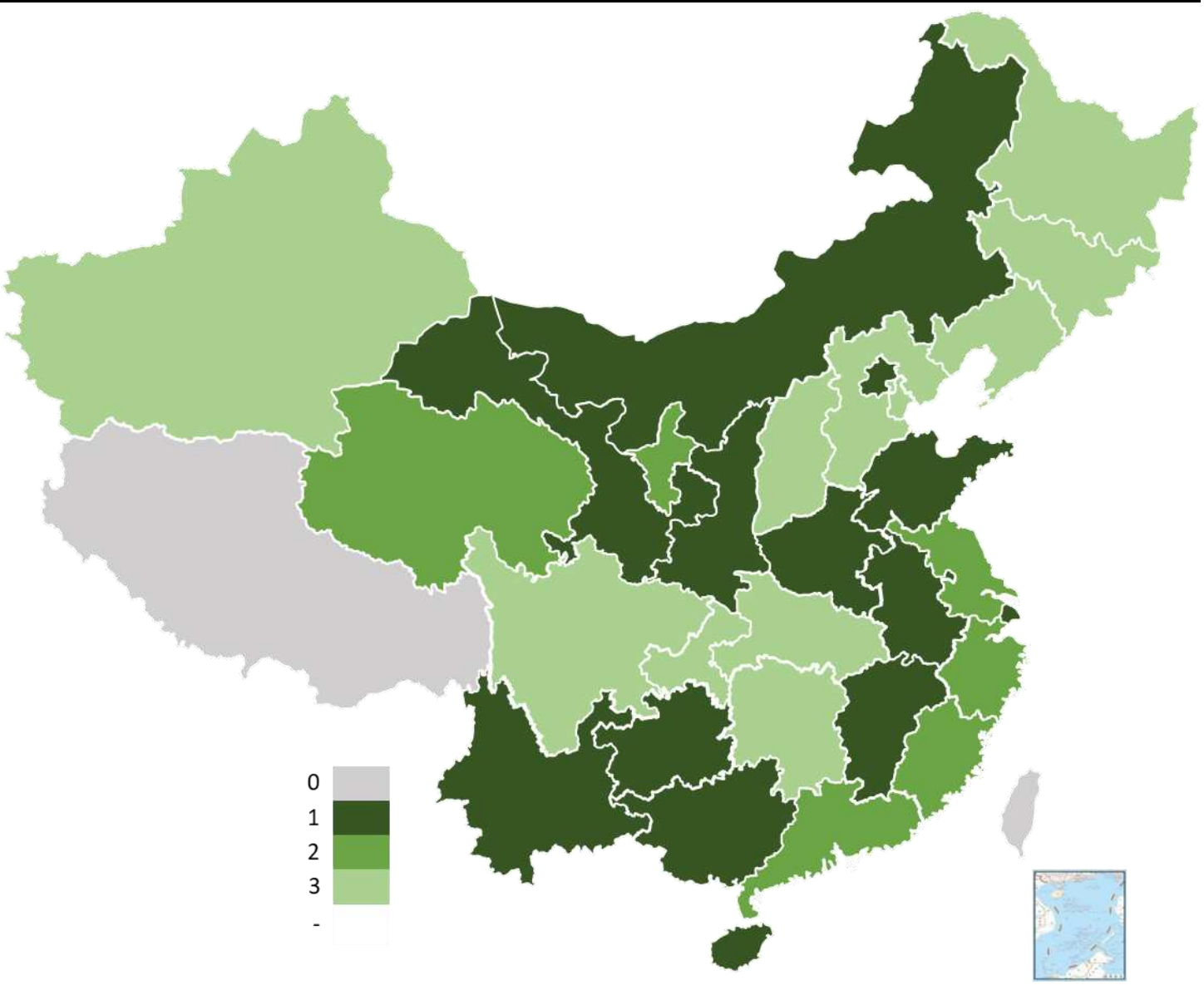

Figure 1 Geographical distribution of electrification rate 
Before conducting econometric model testing, we need to observe the changing trends of variables in each province and each period. The trend chart is as follows, in the threedimensional graph, the $\mathrm{x}$-axis is the province, the $\mathrm{y}$-axis is the year, and the $\mathrm{z}$-axis is the value of each variable. The color changes from blue to yellow, indicating that the value is from small variable value from small to large.

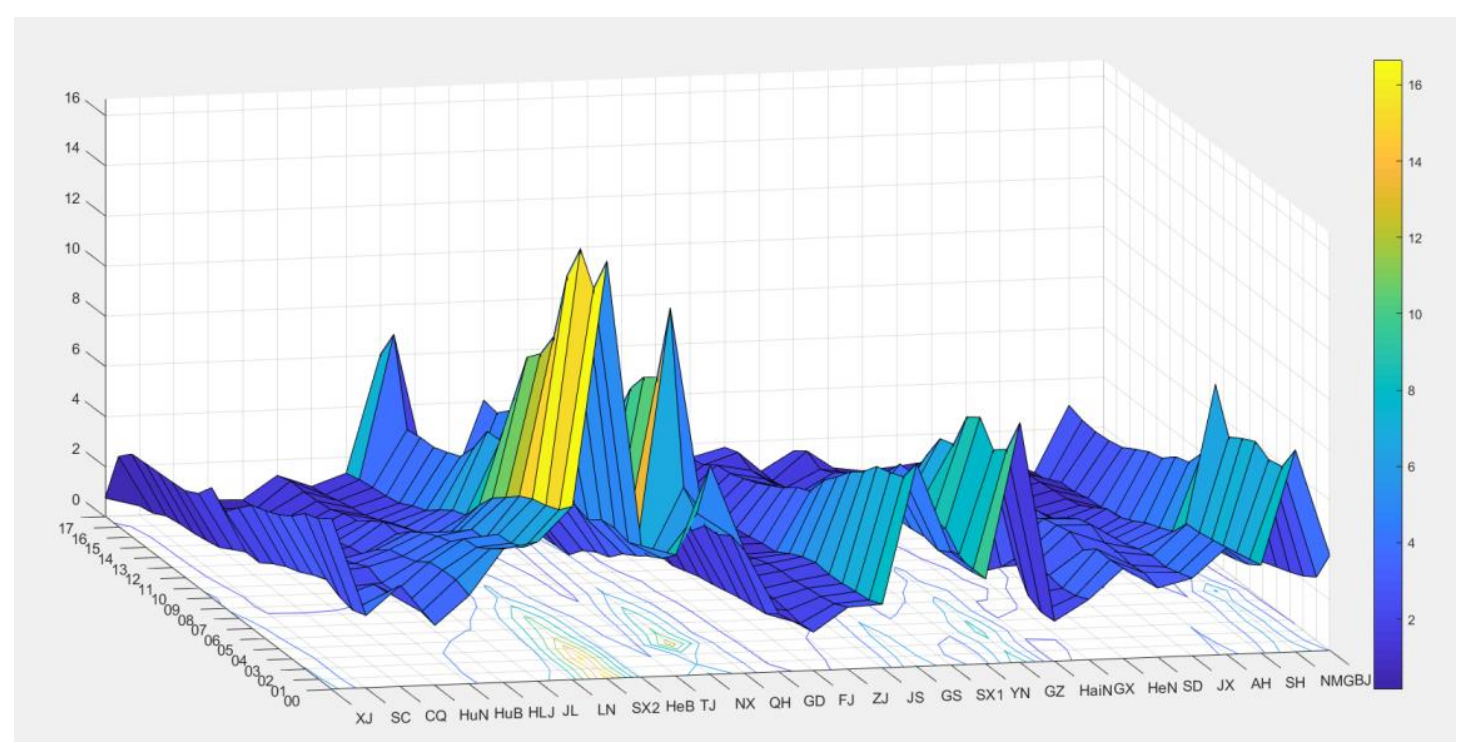

Figure 2 the three-dimensional trend graph of $\mathrm{CI}$ 


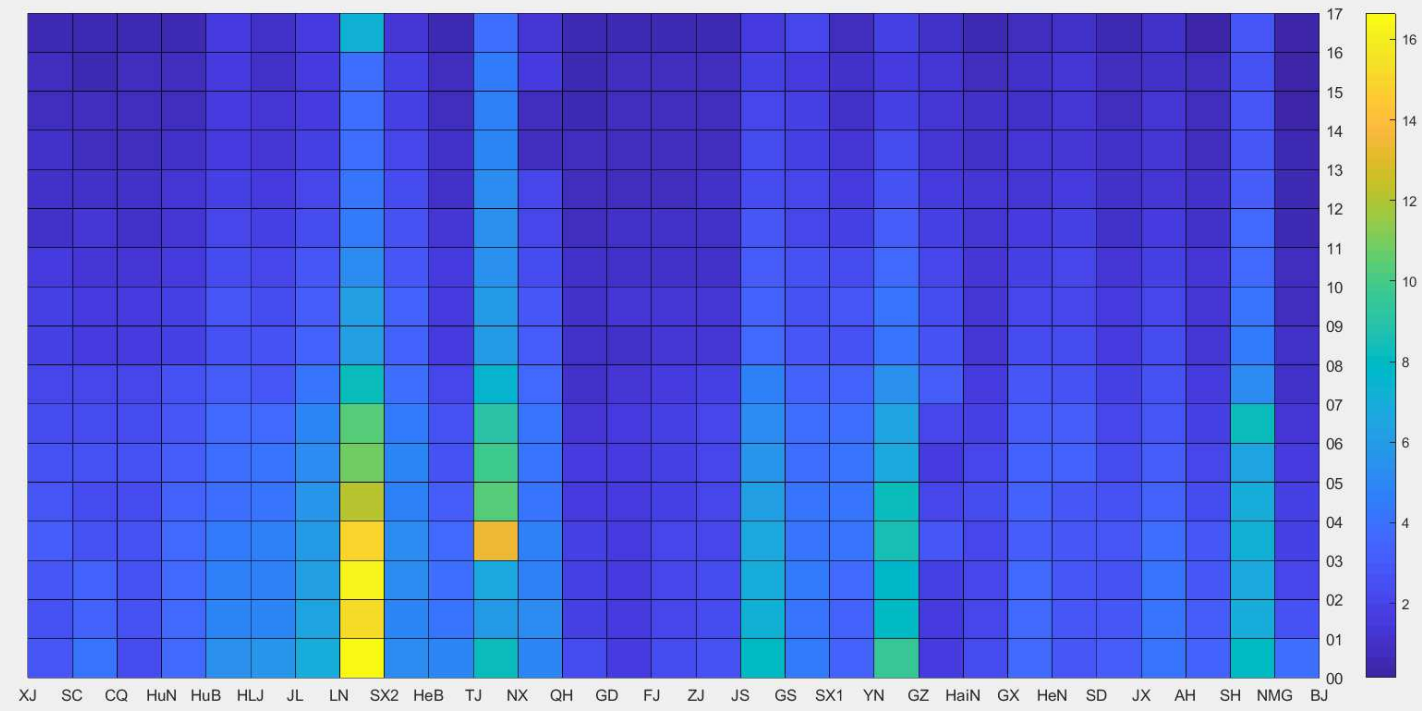

Figure 3 the plane trend graph of $\mathrm{CI}$

Figures 2 and 3 show the three-dimensional trend graph and the flat trend graph of the $\mathrm{CI}$ in

obvious, indicating that $\mathrm{CI}$ has significant differences in the same period in different provinces.

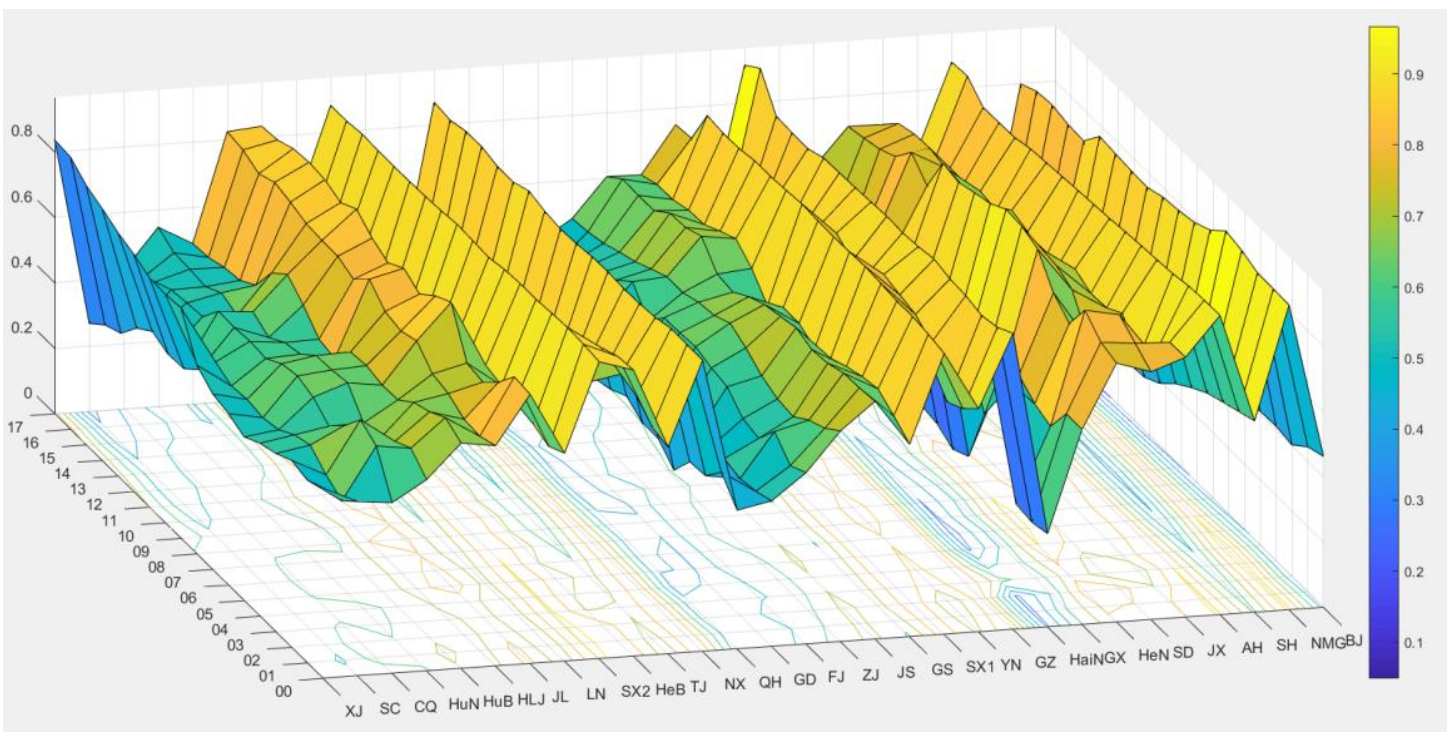




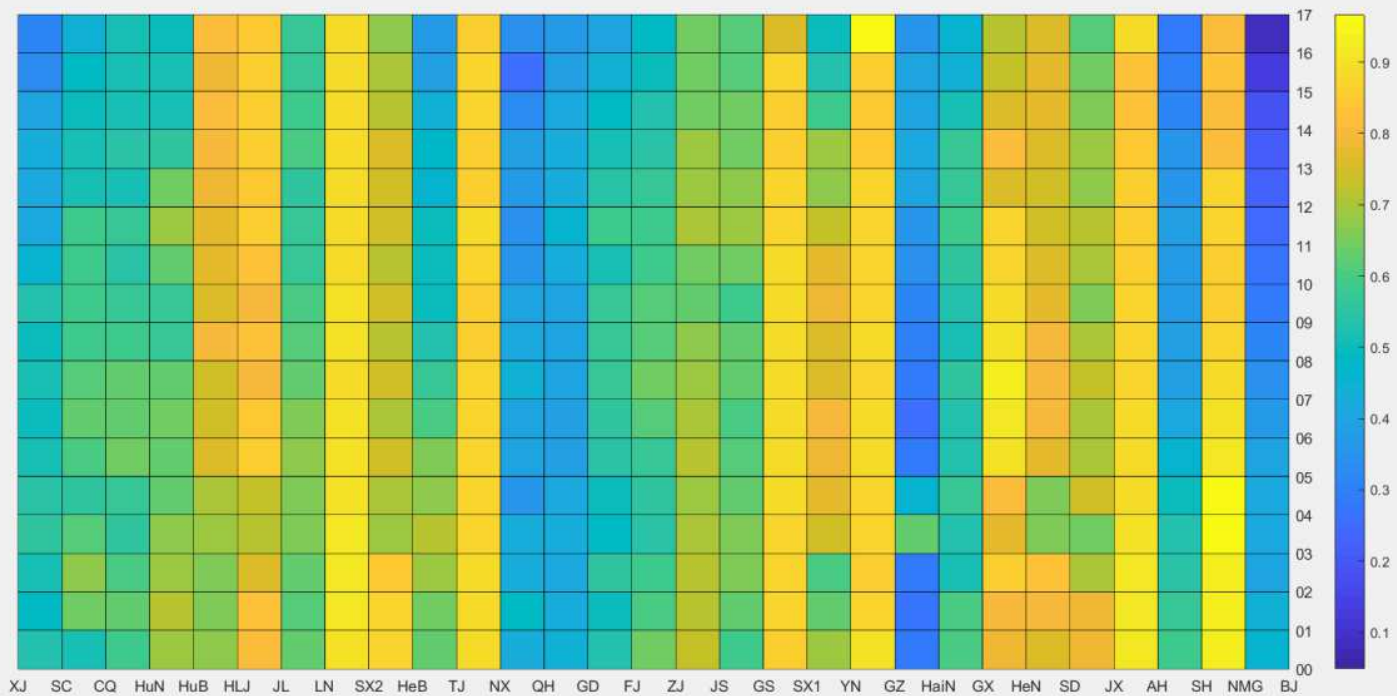

Figure 5 the plane trend graph of ECS

Figures 4 and 5 show the three-dimensional trend graph and the flat trend graph of the ECS

254 in each province from 2000 to 2017. It can be found that the color changes in the figure are 255 obvious, indicating that ECS has significant differences in the same period in different provinces.

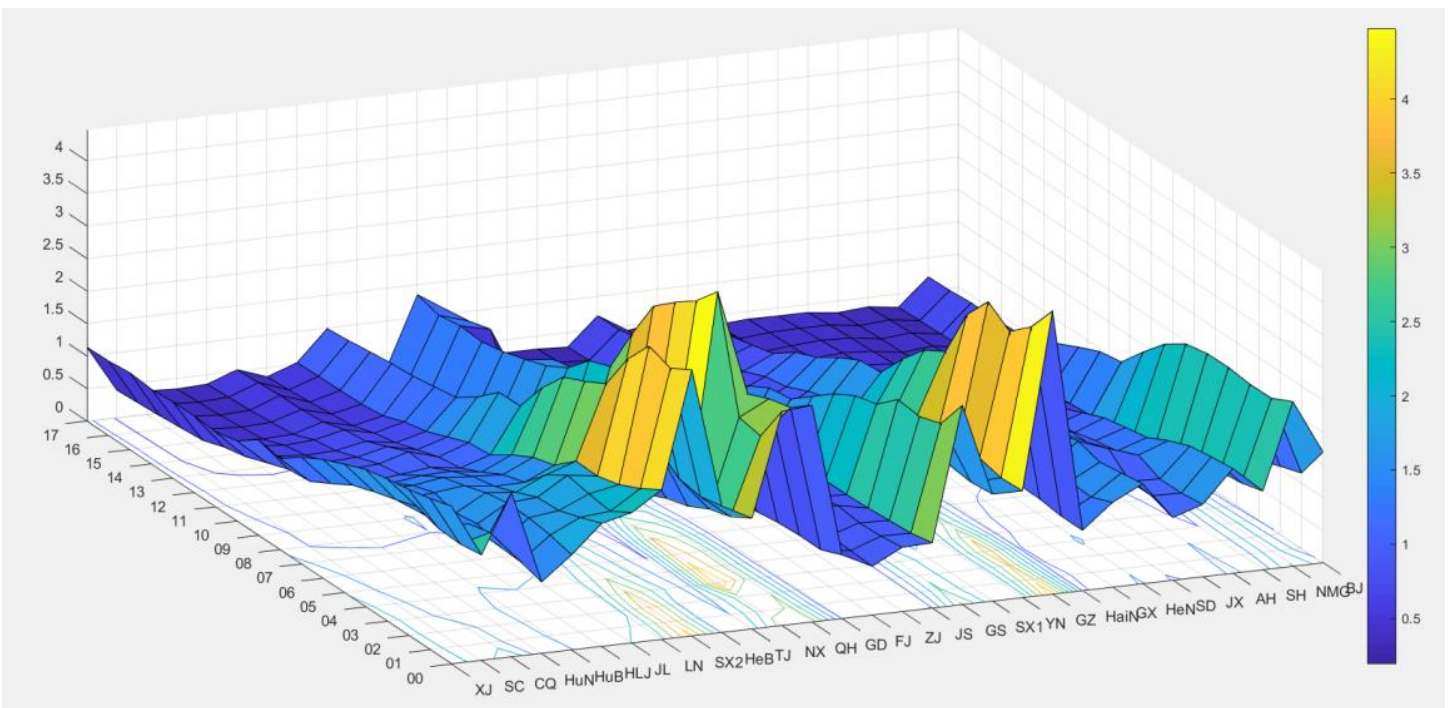




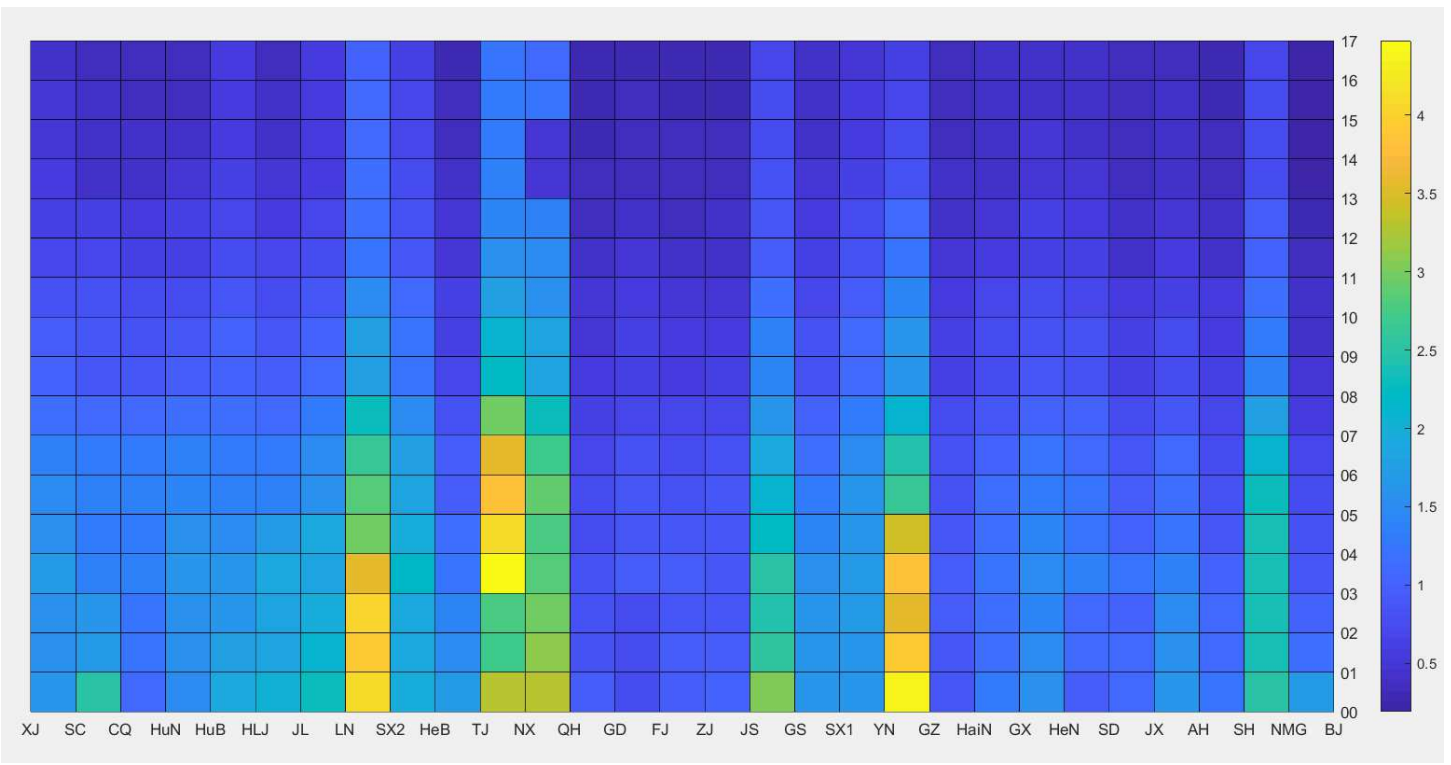

Figure 7 the plane trend graph of EI

Figures 6 and 7 show the three-dimensional trend graph and the flat trend graph of the EI in

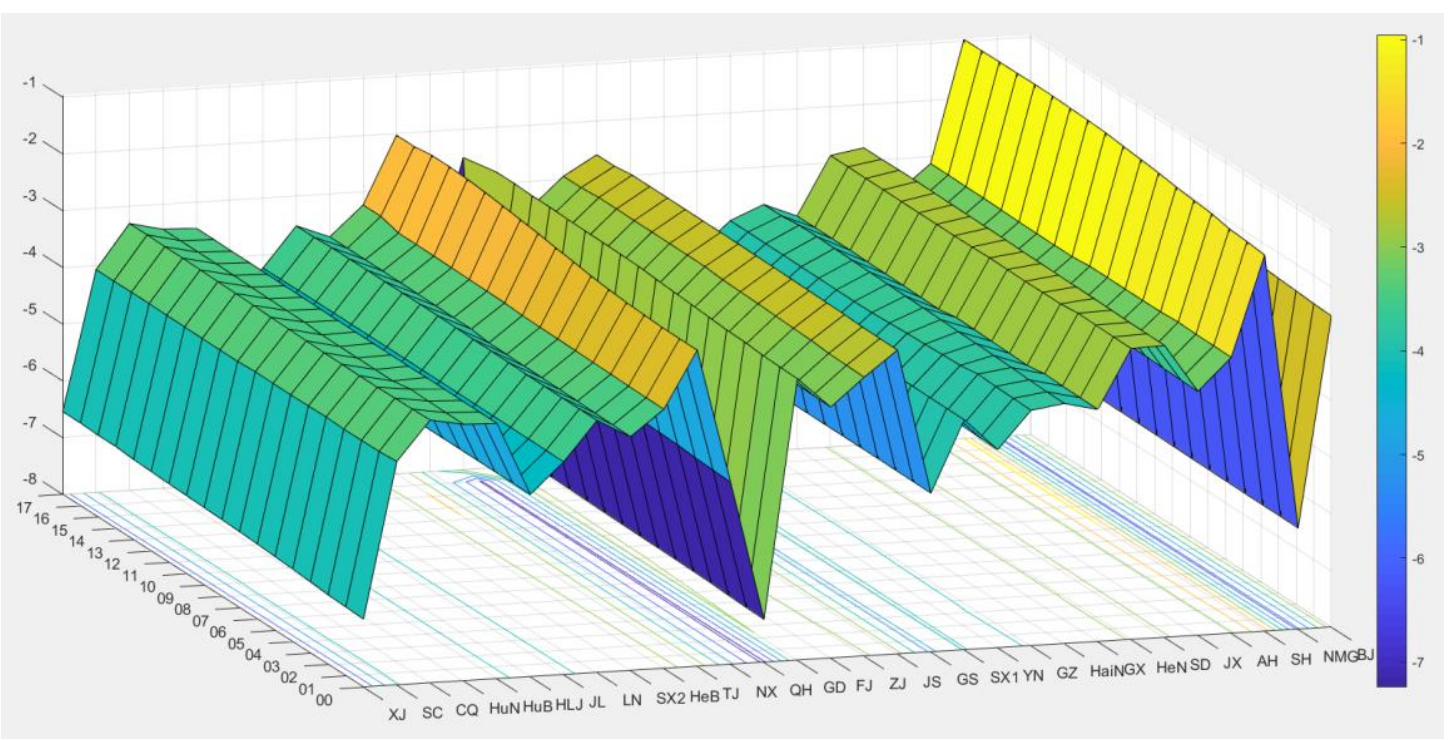

Figure 8 the three-dimensional trend graph of LPD 


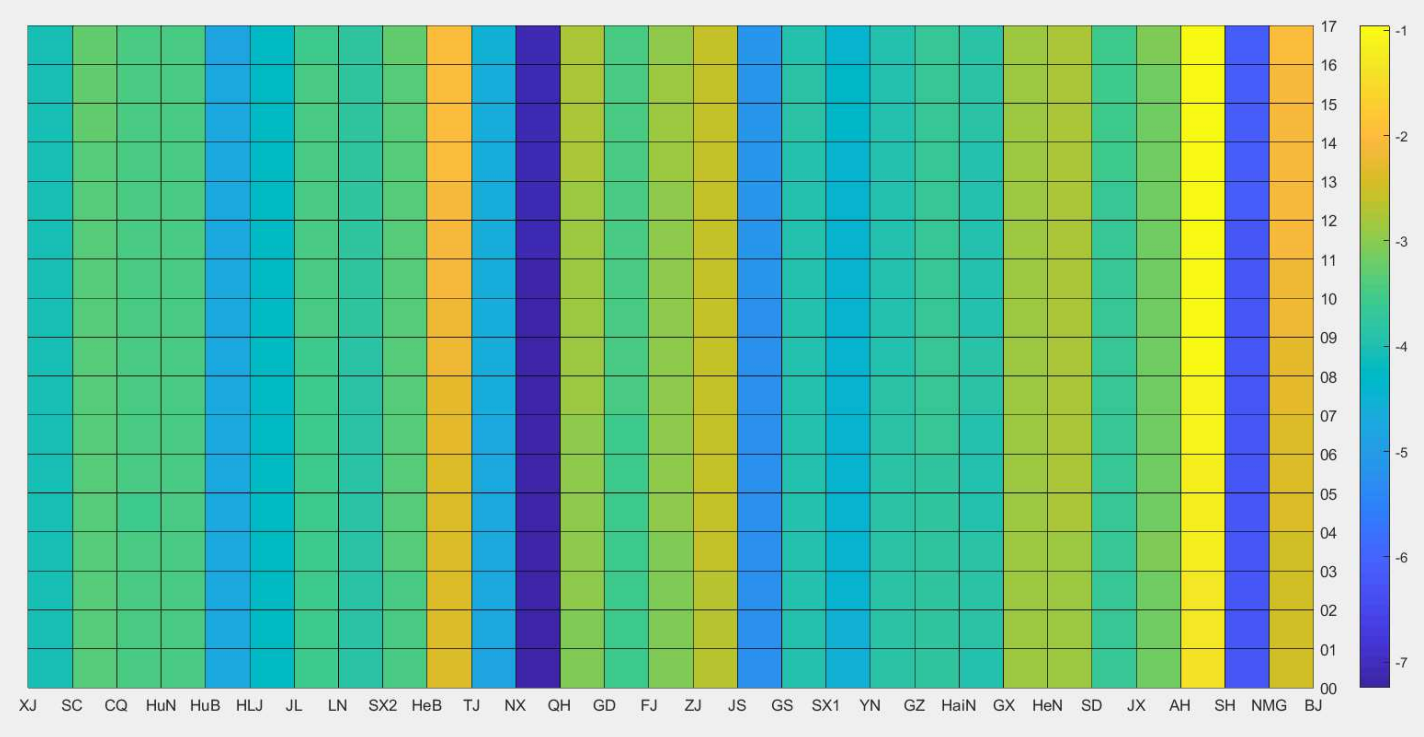

Figure 9 the plane trend graph of LPD

Figures 8 and 9 show the three-dimensional trend graph and the flat trend graph of the LPD

271 in each province from 2000 to 2017. It can be found that the color changes in the figure are 272 obvious, indicating that LPD has significant differences in the same period in different provinces, but the population in each province is relatively stable

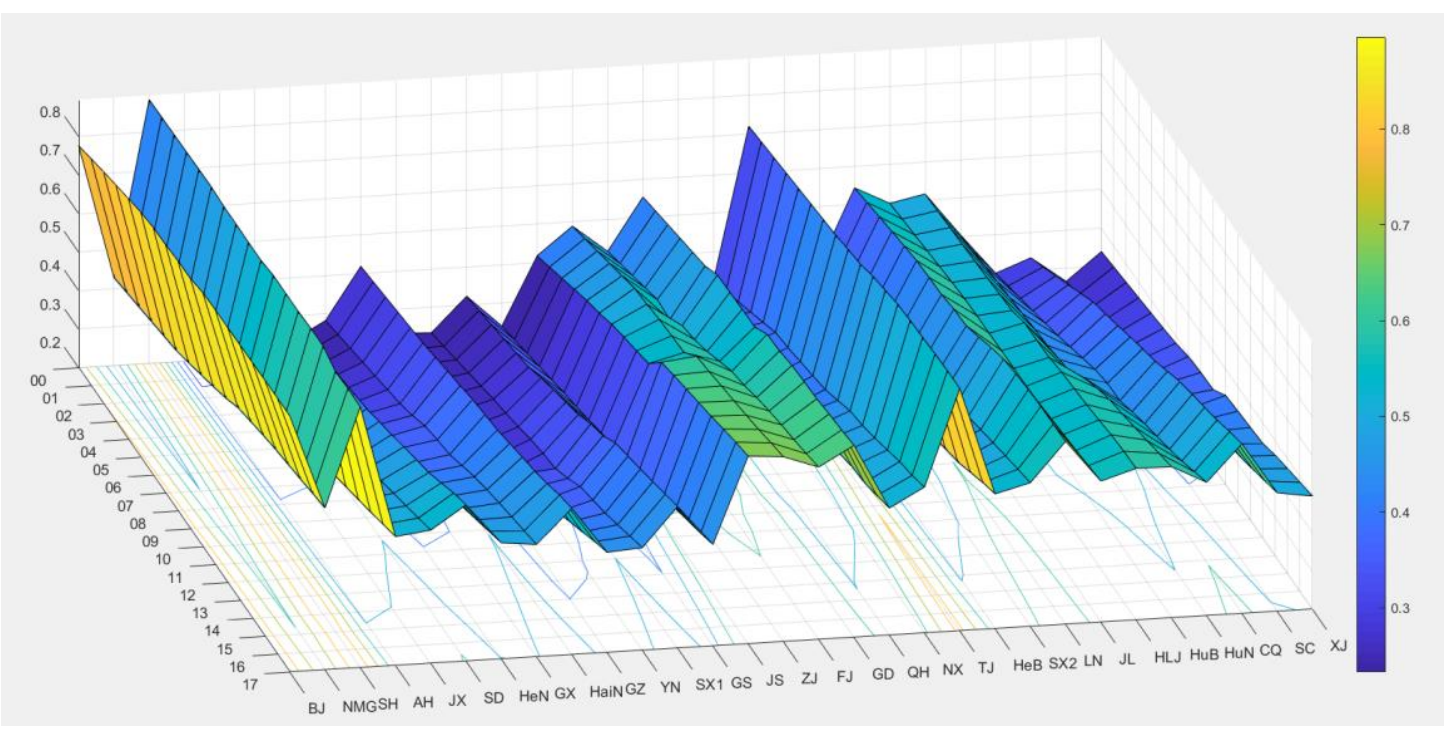

Figure 10 the three-dimensional trend graph of UR 


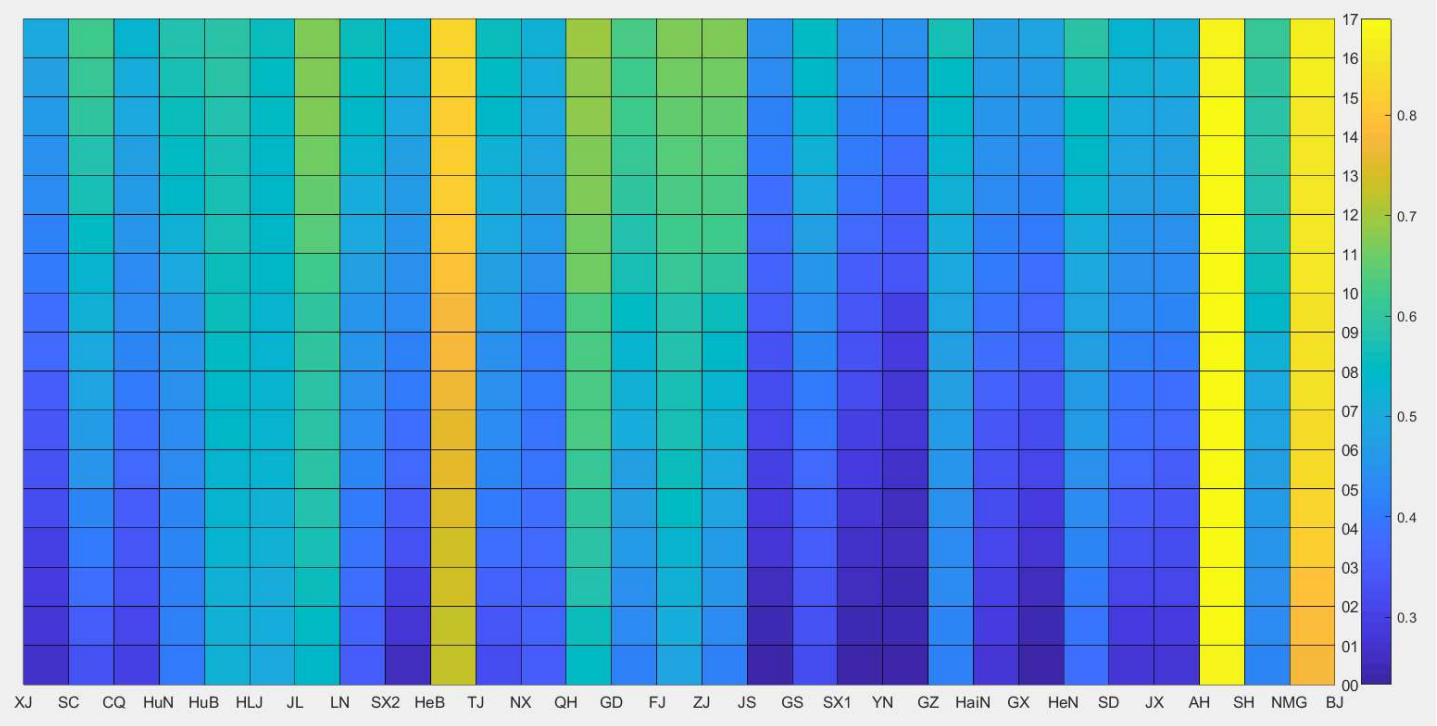

Figure 11 the plane trend graph of UR

Figures 10 and 11 show the three-dimensional trend graph and the flat trend graph of the

281 UR in each province from 2000 to 2017. It can be found that the color changes in the figure are

282 more obvious, indicating that UR has significant differences in the same period in different 283 provinces.

284 In summary, all variables have inter-provincial differences and meet the requirements of

286 Table 2 shows the descriptive statistics of all variables in the national horizontal panel and

287 the three sub-panels. Including mean, median, maximum, minimum, standard deviation,

288 skewness and kurtosis.

Table 2 Descriptive statistics for all variables in panel and cross-sections

\begin{tabular}{|c|c|c|c|c|c|c|c|c|}
\hline \multirow[t]{3}{*}{ Objects } & Variabl & & & & & Std.Dev & Skewne & Kurtosi \\
\hline & & Mean & Median & Max. & Min. & & & \\
\hline & es & & & & & . & ss & $\mathrm{S}$ \\
\hline Nation & CI & 2.8040 & 2.1669 & 16.6536 & 0.1546 & 2.2729 & 2.3578 & 11.5715 \\
\hline
\end{tabular}




\begin{tabular}{|c|c|c|c|c|c|c|c|c|}
\hline & LPD & -3.7795 & -3.5664 & -0.9543 & -7.2422 & 1.2587 & -0.7756 & 4.0326 \\
\hline & EI & 1.1056 & 0.9110 & 4.4755 & 0.1920 & 0.7643 & 1.6896 & 6.3770 \\
\hline & ECS & 0.6447 & 0.6438 & 0.9671 & 0.0491 & 0.1860 & -0.3349 & 2.3580 \\
\hline & UR & 0.5008 & 0.4806 & 0.8960 & 0.2330 & 0.1521 & 0.7968 & 3.3786 \\
\hline \multirow{5}{*}{ HERR } & CI & 2.6085 & 2.1569 & 8.5584 & 0.1546 & 1.7734 & 1.4021 & 4.7450 \\
\hline & LPD & -3.6109 & -3.6851 & -0.9543 & -6.2121 & 1.2338 & -0.0578 & 3.2066 \\
\hline & EI & 1.0181 & 0.8353 & 4.3532 & 0.1920 & 0.6976 & 1.8865 & 7.6041 \\
\hline & ECS & 0.6685 & 0.7191 & 0.9671 & 0.0491 & 0.2133 & -0.7126 & 2.4964 \\
\hline & UR & 0.4812 & 0.4395 & 0.8960 & 0.2330 & 0.1863 & 1.0786 & 3.2017 \\
\hline \multirow{5}{*}{ MERR } & CI & 2.4778 & 1.5989 & 13.4085 & 0.3675 & 2.3730 & 2.0680 & 7.7346 \\
\hline & LPD & -3.9211 & -3.0765 & -2.4986 & -7.2422 & 1.5641 & -1.2415 & 3.1347 \\
\hline & EI & 1.1354 & 0.8386 & 4.4755 & 0.2709 & 0.9806 & 1.5959 & 4.7227 \\
\hline & ECS & 0.5725 & 0.5502 & 0.8920 & 0.2606 & 0.1728 & 0.5022 & 2.1836 \\
\hline & UR & 0.5357 & 0.5396 & 0.6985 & 0.3253 & 0.0982 & -0.2007 & 2.0110 \\
\hline \multirow{5}{*}{ LERR } & CI & 3.2128 & 2.5839 & 16.6536 & 0.3842 & 2.6617 & 2.5492 & 11.9038 \\
\hline & LPD & -3.9016 & -3.5207 & -1.9885 & -6.7999 & 1.0702 & -1.1940 & 4.7036 \\
\hline & EI & 1.1928 & 1.1248 & 4.0972 & 0.3025 & 0.6951 & 1.4054 & 6.1314 \\
\hline & ECS & 0.6560 & 0.6396 & 0.9172 & 0.2688 & 0.1447 & 0.0087 & 2.3562 \\
\hline & UR & 0.5048 & 0.5056 & 0.8292 & 0.2622 & 0.1260 & 0.5964 & 3.1949 \\
\hline
\end{tabular}

290 In table 3 , by referring to the $\mathrm{P}$-value $<1 \%$, presents the correlations among the analyzed

291 variables for national level, which indicates that $C I$ have significant positive correlations with 
293 urbanization rate and population density, the efficiency of public infrastructure is improved,

294 and the contribution of resource utilization efficiency to economic growth is greater than that

295 of carbon emission, which leads to the decrease of carbon intensity. Besides, the results also

296 show that UR has negative correlations with ECS, indicating that a low energy efficiency is

297 harmful to urbanization. And coal consumption is still a significant element promoting GDP

298 and $U R$.

Table 3 Correlations for the panel data set ( $\mathrm{p}$ values in parentheses)

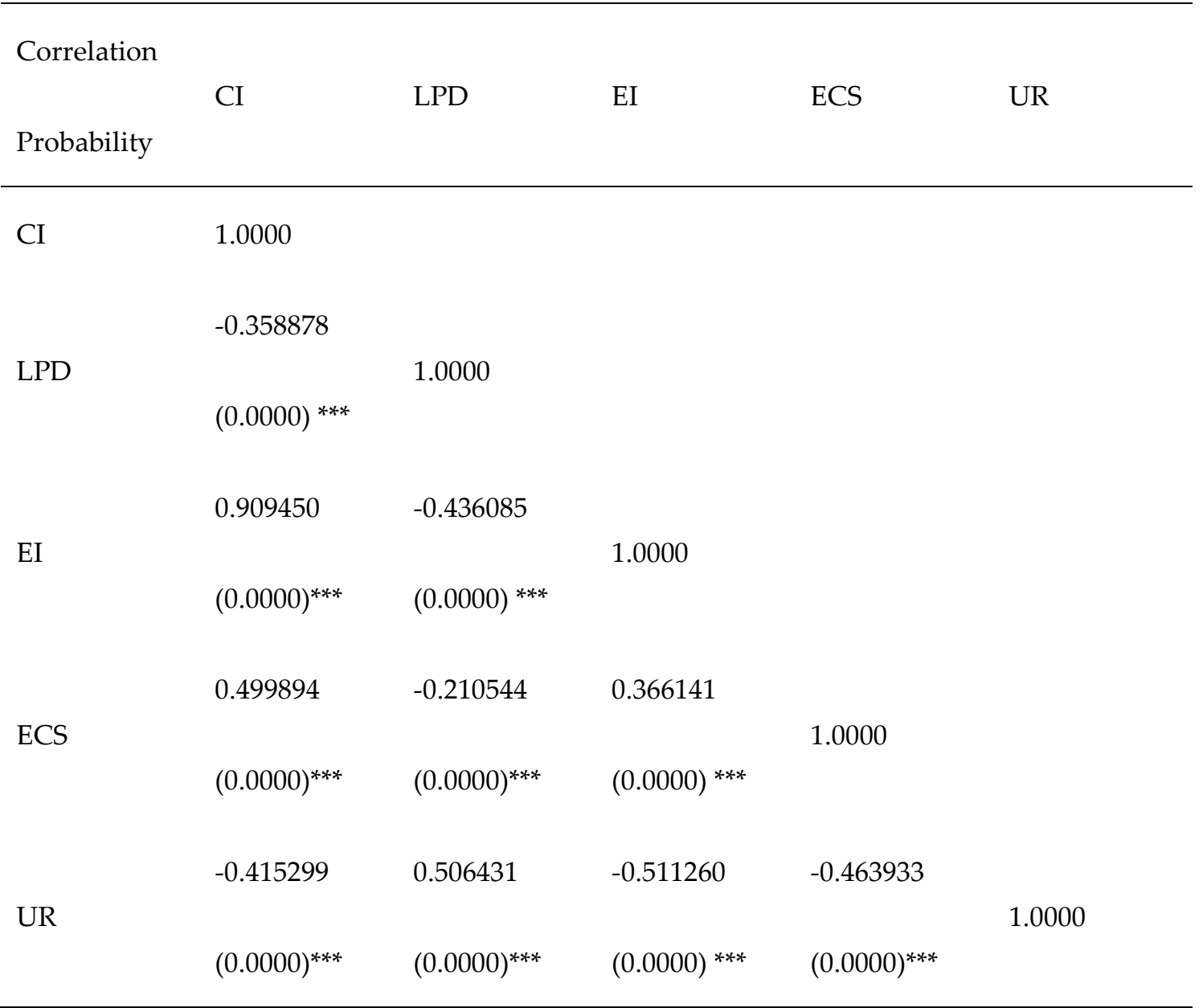

300 Note: *** Denotes statistical significance at $1 \%$ level. 

standard represent the model:

$$
Y_{a t}=\alpha_{a}+\beta_{a t} X_{a t}+\mu_{a t}, \quad a=1,2, \ldots, A, \quad t=1,2, \ldots, T
$$
$\operatorname{cor}\left(\mu_{a \mathrm{t}}, \mu_{\mathrm{t} a}\right) \neq 0$, where $\rho_{\mathrm{ad}}$ is calculated as follow:

317 false regression. According to existing studies(Levin A et al.2002, Breitung J.2001), unit root 318 tests models are widely used for panel unit root tests. However, for panels with cross-sectional 319 dependencies, the first-generation unit root test tends to over-reject the null hypothesis(Pesaran 320 M H.2007, Bhattacharya M et al.2016). Thus, the cross-section Im-Pesaran-Shin (CIPS) method 321 developed by Pesaran(Pesaran, M H,2004) is adopted, using average individual statistics as follows:: 
324 where $\bar{z}_{a}=N^{-1} \sum_{a=1}^{N} z_{a b}$ and $\Delta$ represents the difference operator. Considering the serial correlation in the data, the model can be extended as follows:

$$
\Delta z_{a t}=\alpha_{a}+\beta_{a}^{*} z_{a, t-1}+c_{0} \bar{z}_{a-1}+\sum_{d=0}^{r} c_{d+1} \Delta \bar{z}_{t-d}+\sum_{k=1}^{r} b_{k} \Delta z_{a, t-k}+\mu_{a t}
$$
regression is performed according to (7), and then the $\mathrm{t}$ statistic of $\beta_{i}^{*}$ is obtained.

$$
\text { CIPS }=A^{-1} \sum_{a=1}^{A} C A D F_{a}
$$

The economic convergence is further classified based on the neoclassical economic growth theory, and the test model is put forward accordingly. Convergence analysis can be divided into the following three categories:

\subsection{1 $\alpha$ convergence,}

This paper, the compiler coefficient method in the $\alpha$ convergence model is used to evaluate the development trend of the absolute gap of carbon emission efficiency in regions with different electrification rates in selected 30 provinces.

$$
\bar{X}=\frac{1}{n} \sum_{i=1}^{n} x_{i}
$$

$$
\alpha=\sqrt{\frac{1}{n-1} \sum_{i=1}^{n}\left(X_{i}-\bar{X}\right)^{2}}
$$

$X_{i}$ is the carbon emission intensity of the year under a certain electrification rate level, $\bar{X}$

341 is the mean value of carbon intensity, and $\alpha$ is the standard deviation. If the standard 
deviation shows a decreasing trend in time series, the carbon emission efficiency during this period is $\alpha$ Convergence.

\subsection{2 $\beta$ absolute convergence}

has a tendency to move toward high carbon emission intensity, that is, to judge whether there convergence model is shown below.

$$
\Delta C I_{i}=\alpha+\beta C I_{i, t}+\mu_{i, t}
$$

Where $C I_{i, t}$ indicates the carbon emission intensity at a certain electrification rate level in a

certain year $\Delta C I_{i}$ indicates the difference between the carbon emission intensity at the electrification rate level of $\mathrm{t}+1$ and $\mathrm{t}$ years. $\alpha$ is a constant term. $\mu_{i, t}$ is an error term. When $\beta$ $<0$, the results pass the significance test, it is proved that there is the" catch-up effect" on the carbon emission intensity at the electrification rate level.

\subsection{3 $\beta$ conditional convergence}

$$
\Delta C I_{i}=\alpha+\beta C I_{i, t}+\lambda X_{\mathrm{i}}+\mu_{i, t}
$$


362 Where $C I_{i, t}$ indicates the carbon emission intensity at a certain electrification rate level in a

363 certain year $\Delta C I_{i}$ indicates the difference between the carbon emission intensity at the

364 electrification rate level of $t+1$ and $t$ years. $\alpha$ is a constant term. $\mu_{i, t}$ is an error term. $x_{i}$ is the

365 control variable.

366 Empirical findings and interpretations

367 4.1 Cross-sectional dependence test

368 The results of cross-sectional dependence test is presented in Table 4. It can be seen that five

369 variables, i.e., $C I, L P D, E I$, UR and $E C S$, reject the null hypothesis in all panels within ten

370 percent, which indicates that the cross-sectional dependence exists. Therefore, the second

371 generation of panel unit root detection technology is introduced.

Table 4 Pesaran cross-sectional dependence test results

\begin{tabular}{|c|c|c|c|c|c|c|c|}
\hline Region & Variable & $\mathrm{CI}$ & LPD & EI & ECS & UR & Overall \\
\hline \multirow{4}{*}{ Nation } & Pesaran & & & & & & \\
\hline & & 15.14808 & 10.5524 & 15.82276 & 19.18348 & 4.907672 & -1.899475 \\
\hline & $\mathrm{CD}$ test & & & & & & \\
\hline & Prob. & $0.0000^{* * *}$ & $0.0000^{* * *}$ & $0.0000^{* * *}$ & $0.0000^{* * *}$ & $0.0000^{* * *}$ & $0.0575^{*}$ \\
\hline \multirow{4}{*}{ HERR } & Pesaran & & & & & & \\
\hline & & -2.903043 & -3.03014 & -2.87702 & -2.921711 & -2.997068 & -2.178282 \\
\hline & CD test & & & & & & \\
\hline & Prob. & $0.0037^{* *}$ & $0.0024^{* * *}$ & $0.0040^{* * *}$ & $0.0035^{* * *}$ & $0.0027^{* * *}$ & $0.0294^{* *}$ \\
\hline \multirow[b]{2}{*}{ MERR } & Pesaran & & & & & & \\
\hline & & 15.09503 & 14.49743 & 14.4493 & 15.43831 & 15.29589 & 7.598773 \\
\hline
\end{tabular}


Prob. $\quad 0.0000^{* * *} \quad 0.0000^{* * *} \quad 0.0000^{* * *} \quad 0.0000^{* * *} \quad 0.0000^{* * *} \quad 0.0000^{* * *}$

Pesaran

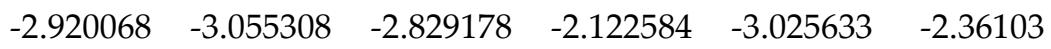

LERR CD test

Prob. $\quad 0.0035^{* * *} \quad 0.0022^{* * *} \quad 0.0047^{* * *} \quad 0.0338^{* *} \quad 0.0025^{* * *} \quad 0.0182^{* *}$

373 Notes: ${ }^{* *},{ }^{* *},{ }^{*}$ Denotes the rejection of null hypothesis at $1 \%, 5 \%, 10 \%$ significance level.

374 4.2 Panel unit root test

375 As described above, because the existence of cross-sectional dependence makes results of

376 first generation panel unit root test methods biased, recently developed second generation

377 panel unit root test technique named CIPS test is adopted in this section to inspect stationarity

378 with CI, LPD, EI, ECS and UR. Results of the panel unit root test is presented in Table 5,

379 indicating that all variables are non-stationary at stage that are non-stationary at their first

380 difference.

Table 5 Panel unit roots results

\begin{tabular}{|c|c|c|c|c|c|c|c|c|c|}
\hline \multirow{3}{*}{ Region } & \multicolumn{3}{|c|}{ Variable } & & \multicolumn{5}{|c|}{ Criterion } \\
\hline & \multirow{3}{*}{ test } & \multirow{3}{*}{ CI } & \multirow{3}{*}{ LPD } & \multirow{3}{*}{ EI } & \multirow{3}{*}{ ECS } & \multirow{3}{*}{ UR } & $10 \%$ & \multirow{3}{*}{$5 \%$ level } & \multirow{3}{*}{$1 \%$ level } \\
\hline & & & & & & & & & \\
\hline & & & & & & & level & & \\
\hline \multirow[t]{2}{*}{ Nation } & Level & -1.705 & -0.58 & $-3.051^{* * *}$ & -2.092 & -1.643 & -2.11 & -2.2 & -2.38 \\
\hline & 1st diff. & $-3.558^{* * *}$ & -0.958 & - & $-3.608^{* * *}$ & -2.334 & -2.11 & -2.2 & -2.38 \\
\hline \multirow[t]{2}{*}{ HERR } & Level & -1.792 & -1.189 & $-2.735^{* * *}$ & -1.984 & -1.459 & -2.11 & -2.22 & -2.45 \\
\hline & 1st diff. & $-3.495^{* * *}$ & $-2.901^{* * *}$ & - & $-3.678^{* * *}$ & $-2.748^{* *}$ & -2.22 & -2.4 & -2.76 \\
\hline
\end{tabular}




\begin{tabular}{|c|c|c|c|c|c|c|c|c|c|}
\hline MERR & Level & -2.106 & -0.218 & -2.091 & $-2.286^{*}$ & -1.683 & -2.18 & -2.33 & -2.64 \\
\hline \multirow{3}{*}{ LERR } & 1st diff. & $-3.844^{* * *}$ & -0.732 & $-4.221^{* * *}$ & $-4.167^{* * *}$ & $-3.611^{* * *}$ & -2.15 & -2.29 & -2.56 \\
\hline & Level & $-2.459^{* * *}$ & -1.661 & $-3.364^{* * *}$ & $-2.138^{*}$ & $-2.311^{* *}$ & -2.11 & -2.22 & -2.45 \\
\hline & 1st diff. & - & $-2.983^{* * *}$ & - & $-3.637^{* * *}$ & - & -2.11 & -2.22 & -2.45 \\
\hline
\end{tabular}

382 Notes: ${ }^{*}$ CIPS test is estimated applying constant and trend with 1 lag.

$383 * * * * * *$ Denotes the rejection of null hypothesis at 1\%, 5\%, $10 \%$ level of significance.

$384 \quad 4.3$ Convergence analysis results

$385 \quad 4.3 .1 \alpha$ convergence results

386 In this paper, the carbon intensity from 2000 to 2015 has been tested for $\alpha$ convergence, and 387 the standard deviation of each year is shown in Table 6 at the level of Nation, HERR, MERR 388 and LERR:

Table 6: standard deviation under Nation, HERR, MERR, LERR level

\begin{tabular}{ccccc}
\hline Year & Nation & HERR & MERR & LERR \\
\hline 2000 & 3.018154 & 2.774374 & 2.934958 & 3.638367 \\
2001 & 2.996758 & 2.616011 & 2.698242 & 3.432976 \\
2002 & 3.009933 & 2.707337 & 2.717034 & 3.497186 \\
2003 & 3.110857 & 2.763777 & 3.478131 & 3.336141 \\
2004 & 2.874087 & 2.678702 & 3.05058 & 3.045049 \\
2005 & 2.771108 & 2.556362 & 3.002436 & 2.93052 \\
2006 & 2.716868 & 2.571348 & 2.896845 & 2.831414
\end{tabular}




\begin{tabular}{lllll}
2007 & 2.48737 & 2.339352 & 2.662771 & 2.602084 \\
2008 & 2.290414 & 2.176027 & 2.451697 & 2.372895 \\
2009 & 2.276582 & 2.155688 & 2.428462 & 2.370804 \\
2010 & 2.178551 & 2.073204 & 2.335825 & 2.251123 \\
2011 & 2.114532 & 2.006879 & 2.317713 & 2.162923 \\
2012 & 2.073267 & 1.961625 & 2.281376 & 2.122359 \\
2013 & 2.022632 & 1.914733 & 2.201831 & 2.082521 \\
2014 & 1.995876 & 1.885246 & 2.164931 & 2.06381 \\
2017 & 1.98808 & 1.871111 & 2.178626 & 2.051068 \\
2016 & 2.023763 & 1.858509 & 2.129298 & 2.183571 \\
2017 & 1.898822 & 1.843966 & 1.746923 & 2.066937 \\
\hline
\end{tabular}

391 table is converted into chart form, as shown in figure 12.

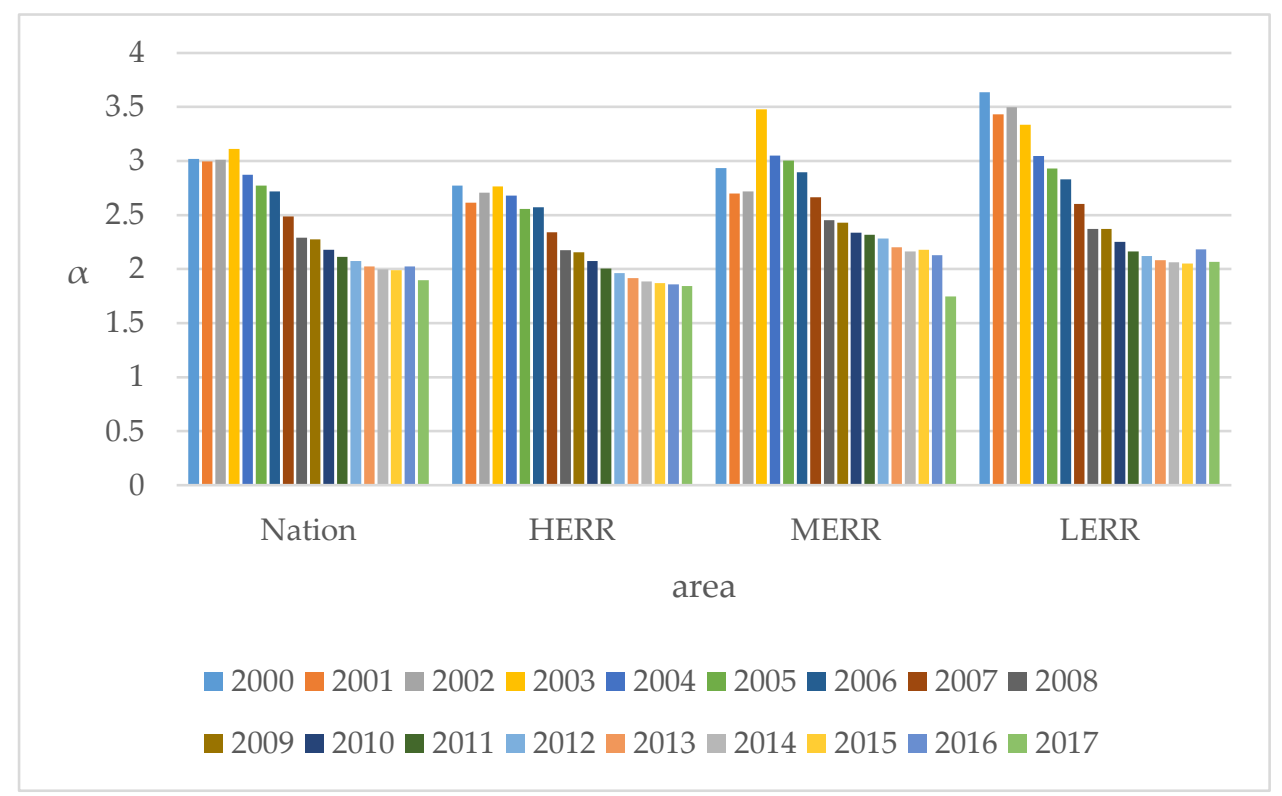

393 Figure 12 development trend of carbon emission intensity standard deviation in time series. 

monotonous decline, indicating that China's carbon emission control level has significantly

396 improved and the carbon emission intensity has continued to decline.

397 The HERR panel data is lower than the national level as a whole, while LERR is higher than 398 the national level as a whole.

399 This is mainly due to the mature economic development in areas with high electrification 400 rate and relatively stable economic growth. The energy structure transformation process is 401 relatively fast, and the energy consumption structure is relatively optimized. Therefore, the 402 degree of fluctuation of carbon intensity is small. In areas with low electrification rates, the 403 energy structure is still dominated by coal power, the transformation of the energy structure is 404 intensified, and economic growth is also fast, so the volatility of carbon intensity is relatively 405 high.

406 The overall degree of fluctuation in various regions tends to decline, but the carbon emission 407 intensity increased significantly in 2003, which requires further study.

\begin{tabular}{lccccc}
\hline \multirow{2}{*}{ Region } & \multicolumn{5}{c}{ LS } \\
\cline { 2 - 6 } & Coefficient & Std. Error & t-Statistic & Prob. & R-squared \\
\hline \multirow{2}{*}{ Nation } & -0.6765 & 0.0403 & -16.7839 & 0.0000 & 0.3567 \\
& -0.8168 & 0.0686 & -11.9146 & 0.0000 & 0.3933
\end{tabular}




$\begin{array}{llllll}\text { MERR } & -0.7245 & 0.0470 & -15.4176 & 0.0000 & 0.3188 \\ \text { LERR } & -0.5587 & 0.0698 & -8.0000 & 0.0000 & 0.2570\end{array}$

410 As can be seen from Table 7, coefficient are all negative in the regression results of Nation,

411 HERR, MERR, and LERR panels, and the P value is less than 0.01 , which indicates there is a

412 "catch-up effect " in the carbon intensity under the horizontal panel of each electrification rate,

413 the carbon intensity is absolutely convergent.

$414 \quad 4.3 .3 \beta$ conditional convergence results

\begin{tabular}{|c|c|c|c|c|c|}
\hline \multirow{2}{*}{ Region } & \multirow{2}{*}{ Variable } & \multicolumn{3}{|c|}{ LS } & \multirow[b]{2}{*}{ Prob. } \\
\hline & & Coefficient & Std. Error & t-Statistic & \\
\hline \multirow[t]{6}{*}{ Nation } & CI & -0.9976 & 0.1076 & -9.2750 & 0.0000 \\
\hline & LPD & 0.3803 & 0.0839 & 4.5351 & 0.0000 \\
\hline & EI & 1.6134 & 0.3187 & 5.0626 & 0.0000 \\
\hline & ECS & -3.6350 & 0.6088 & -5.9707 & 0.0000 \\
\hline & UR & -2.024382 & 0.7666 & -2.6408 & 0.0085 \\
\hline & R-squared & \multicolumn{4}{|c|}{0.4735} \\
\hline \multirow[t]{4}{*}{ HERR } & $\mathrm{CI}$ & -2.0125 & 0.1140 & -17.6478 & 0.0000 \\
\hline & LPD & -0.3297 & 0.0541 & -6.0925 & 0.0000 \\
\hline & EI & 3.4861 & 0.2741 & 12.7181 & 0.0000 \\
\hline & ECS & -1.5924 & 0.3709 & -4.2929 & 0.0000 \\
\hline
\end{tabular}


UR

$-1.998095$

0.3128

6.3878

0.0000

R-squared

0.6486

\begin{tabular}{|c|c|c|c|c|c|}
\hline \multirow[t]{6}{*}{ MERR } & CI & -2.1198 & 0.0972 & -21.8156 & 0.0000 \\
\hline & LPD & -0.3977 & 0.0501 & -7.9455 & 0.0000 \\
\hline & EI & 3.7124 & 0.2331 & 15.9279 & 0.0000 \\
\hline & ECS & -2.9422 & 0.4609 & -6.3837 & 0.0000 \\
\hline & UR & 2.1159 & 0.4323 & 4.8948 & 0.0000 \\
\hline & R-squared & \multicolumn{4}{|c|}{0.8198} \\
\hline \multirow[t]{6}{*}{ LERR } & CI & -1.9640 & 0.1542 & -12.7404 & 0.0000 \\
\hline & LPD & 0.8545 & 0.1331 & 6.4199 & 0.0000 \\
\hline & EI & 5.3668 & 0.5619 & 9.5514 & 0.0000 \\
\hline & ECS & 5.5659 & 1.0856 & 5.1270 & 0.0000 \\
\hline & UR & -0.8567 & 0.9085 & -0.9430 & 0.3469 \\
\hline & R-squared & \multicolumn{4}{|c|}{0.511888} \\
\hline
\end{tabular}

416

417 As can be seen from Table 8 , in the $\beta$ conditional convergence regression results, $\beta<0$ and

418 passed the significance test, that is, in regions with different levels of electrification rates, the

419 carbon intensity approaches their respective steady-state levels. The energy consumption

420 structure, population density, energy intensity will positively promote carbon intensity, while

421 the urbanization rate in the HERR panel has a negative inhibitory effect on carbon intensity at

422 the level of $10 \%$. In other panels, the urbanization rate does not affect carbon intensity 
significant impact.

\section{Conclusions and policy implications}

As the largest carbon dioxide emitter, China has solemnly promised to reduce carbon

emissions. However, as a developing country, china's task of reducing carbon is arduous,

because a large amount of fossil energy is necessary to fuel the rapid economic development.

It should be noted that different levels and methods of economic development in different

regions of China make it difficult to implement a unified carbon reduction policy. In addition,

the acceleration of China's urbanization process also has an impact on carbon intensity, and

this effect is different in various regions. Therefore, in the context of urbanization, exploring practically important.

434 Data clustering based on the electrification rate is employed in this paper to classify 30

435 provinces of China into three regions. The heterogeneous panel analysis technology and $\alpha, \beta$

436 convergence analysis are used to examine the relationship between energy intensity, energy

437 consumption structure, population density, urbanization rate and carbon intensity. These

438 results obtained adopted a more objective approach to reveal the factors that affect the carbon

439 intensity of China's current socio-economic environment and provided theoretical basis and

440 empirical support for the formulation of carbon reduction policies targeted at different regions.

441 This paper uses heterogeneous panel technology and convergence analysis technology to

442 study the influencing factors of regional carbon intensity in China. Main findings are as follows:

443 (1) According to the results of $\alpha$ convergence analysis and $\beta$ convergence analysis, in Nation, 

indicates that there is a "catch-up effect". Also, it approaches the respective steady-state levels. This means that the national electrification development has become more balanced, and the

447 level of electrification in Nation panel will continue to increase. The electrification process will more effectively promote the green and low-carbon transformation of energy and power development, and provide strong supports for economy development.

(2) The result of $\beta$-condition convergence shows that in the heterogeneous panels, there is a long-term equilibrium relationship between carbon intensity and population density, energy intensity, and energy consumption structure. In the national panel, population density and energy intensity all have a positive effect on the growth of carbon intensity. Among them, energy intensity plays a more significant role, while the urbanization rate and energy consumption structure have a significant inhibitory effect on the growth of carbon intensity. In

456 areas with high and medium electrification rates, energy intensity promotes the growth of 457 carbon intensity most significantly, followed by urbanization rate, and energy consumption 458 structure has a significant inhibitory effect on the growth of carbon intensity. In areas with low 459 electrification rates, energy consumption structure and energy intensity have a significant role 460 in promoting carbon intensity growth, but population density has an inhibitory effect on 461 carbon intensity growth. The main reason for this is that regions with higher electrification 462 levels have more advanced energy-saving and emission-reduction technologies. Energy-saving 463 and emission-reduction technologies have improved energy efficiency, thereby reducing 464 energy consumption and suppressing carbon intensity. 

characterized by the traditional economic development mode of "three highs and one low", efficiency, which will bring serious negative impacts on the environment harmony and ecology. model based on oil and gas has not been completed yet, and it is facing the third wave of energy revolution represented by the change in energy utilization mode (Stan.2018). in this context,

480 the urbanization rate has a significant role in promoting carbon intensity.

481 Thirty selected provinces of China are divided into three sub-panels (high electrification rate, 482 medium electrification rate and low electrification rate) according to the electrification rate 483 standard in this paper, rather than based on geographical location in the conventional models.

484 Through empirical analysis, the results of each sub-panel are different from those of the 
comprehensively reflect the relationship between regional carbon intensity, population density, energy consumption structure, energy intensity and urbanization at different levels of economic development. This will provide an empirical reference for the formulation of energy, economic and environmental policies that are more in line with the actual conditions of each region. This is also the practical value of this article.

Based on the finds in this paper, the following policy recommendations for reducing carbon intensity can be proposed:

(1) In regard to the areas with high electrification rates, the empirical results show that energy intensity has the greatest promotion effect on carbon intensity, and energy consumption structure has a significant inhibitory effect on the growth of carbon intensity. It is worth noting that the urbanization rate also has a significant inhibitory effect on carbon intensity. Therefore, the economic development in high electrification areas is becoming more mature, and the energy structure transition process is fast. On the premise of maintaining stable economic growth, the requirements to continue to optimize the energy consumption structure and reduce carbon emissions are particularly important in these areas. Specifically, the government should establish incentives for carbon reduction behaviors in the daily lives of residents to establish a low-carbon, green, and environmentally-friendly consumption concept and lifestyle concept; in addition, it should deepen the transformation of the energy structure and optimize the energy consumption structure according to the national development strategy. At the same time, we should learn from the international experience of cities with a relatively high electrification rate to achieve low-carbon development and a green economy. 
(2) In regard to the areas with a medium electrification rate, the empirical results show that energy intensity and urbanization rate have a significant role in promoting carbon intensity, and energy consumption structure has a restraining effect on the growth of carbon intensity. It shows that the region is still in a "high-input, high-consumption, high-pollution" economic

511 growth model, which is unsustainable. Therefore, in addition to enhancing people's awareness of environmental protection and advocating green and low-carbon living, the industrial upgrading should take the core role to reduce the energy intensity. At the same time, in the 514 process of urbanization, we should promote low-carbon green methods to achieve high-quality 515 economic and urban development.

(3) In regard to the areas with a low electrification rate, energy consumption structure and energy intensity have a significant role in promoting carbon intensity, while the impact of urbanization rate on carbon intensity is not significant. China's economic development has 519 entered the mid-stage of industrialization. The rapid economic development and the heavy520 duty characteristics of the economic structure have led to a strong increase in energy demand, 521 which has brought great challenges to energy supply and environment. Optimizing energy 522 consumption structure and improving energy efficiency have become key factors in coping 523 with energy supply pressure. In low-electrification areas, increasing the level of electrification 524 can effectively promote the improvement of energy efficiency. In combination with the 525 characteristics of China's coal-based energy resources, the focus of optimizing the energy 526 consumption structure should be to vigorously promote the conversion of coal to electricity, 527 and to increase the power in the terminal energy In order to promote the improvement of 
energy efficiency, reduce the total energy consumption and carbon intensity, and reduce environmental pollution.

(4) Overall, there are significant spatial differences in the impact of urbanization rate on

531 carbon intensity in different regions. This is caused by unbalanced development in China, e.g.,

532 urban and rural development unbalances, regional development unbalances, structural

533 unbalances, economic development and real economic development, and insufficient

534 innovation capacity. Promoting a more balanced and comprehensive development can be

535 carried out from three aspects: First, effective fiscal policy, through tax adjustments, should be

536 established to encourage the industrial upgrading of enterprises in low- and medium-

537 electrification regions, in order to transform the economic growth model, reduce carbon

538 intensity, and reduce environmental pollution. The second is to carry out policy interventions

539 in areas with low and medium electrification rates to support its economic development and

540 energy structure transformation. The third is to increase the electrification rate in areas with

541 low electrification rates, vigorously promote the conversion of coal to electricity, and increase

542 the proportion of electricity consumed in terminal energy, in order to promote energy

543 efficiency and reduce carbon intensity.

544 (5) It could be useful to incorporate the improvement of the level of electrification in the

545 whole society into the national energy strategy, gather consensus on electrification

546 development of all parties, and clarify electrification development as an important path to

547 promote energy consumption, environmental reform, and economic development. The

548 government should guide the rational layout and coordinated development of various types 
549 of clean power generation, improve the safe operation and smart level of the power system,

550 increase the power replacement and energy efficiency improvement in the industrial,

551 construction, and transportation fields, deepen the reform of the power system, and stimulate

552 new momentum for electrification development To narrow the gap in electrification levels

553 between different industries and regions. The truly electrified development with green, safe,

554 efficient and intelligent content will promote China's energy production and consumption

555 revolution, supports the coordinated development of the economy and society, promotes the

556 continuous improvement of the ecological environment, and helps the people's quality of life

557 continue to improve.

558 Acknowlegements/Funding

559 This work was supported by the 2018 Key Projects of Philosophy and Social Sciences

560 Research, Ministry of Education, China (grant number 18JZD032) 《Research on constructing

561 energy system policy and mechanism with characteristics of clean, low-carbon emission, safe

562 and high-efficiency》.

563 Credit author statement

564 I have made substantial contributions to the conception or design of the work; or the

565 acquisition, analysis, or interpretation of data for the work.

566 And I have drafted the work or revised it critically for important intellectual content; And

567 have approved the final version to be published; And agree to be accountable for all aspects of 
569 are appropriately investigated and resolved.

570 All persons who have made substantial contributions to the work reported in the manuscript,

571 including those who provided editing and writing assistance but who are not authors, are

572 named in the Acknowledgments section of the manuscript and have given their written

573 permission to be named.

574 No conflict of interest exits in the submission of this manuscript, and manuscript is approved

575 by all authors for publication. I would like to declare on behalf of my co-authors that the work

576 described was original research that has not been published previously, and not under 577 consideration for publication elsewhere, in whole or in part. All the authors listed have 578 approved the manuscript that is enclosed.

\section{Ethical Approval}

580 My manuscript does not report on or involve the use of any animal or human data or tissue, $581 \quad$ Not applicable.

\section{Consent to Participate}

$583 \quad$ Not applicable.

\section{Authors Contributions}

585 As the instructor, Jingqi Sun provided guidance on research ideas and methods. Xiaohui Guo 586 conducted main writing work and empirical research. Yuan Wang wrote some literature 587 reviews and subsequent revisions. Jing Shi provided methodological guidance and 
improvement. Follow-up proofreading and modification were carried out on Yiquan Zhou.

589 Shen Boyang's main job is to proofread and polish the English

590

591 Economic Modelling. 2015(4): 97-103.

602 Anser Muhammad Khalid,Usman Muhammad,Godil Danish Iqbal,Shabbir Malik

603 Shahzad,Sharif Arshian,Tabash Mosab Iqbal,Lopez Lydia Bares. Does globalization affect the

604 green economy and environment? The relationship between energy consumption, carbon 605 dioxide emissions, and economic growth.[J]. Environmental science and pollution research 605 dioxide emissions, and economic growth.[J]. Environmental science and pollution research 607 Union Nations, 1960-2025. Social science research, 2007, 36(3): 855-872.

\section{Availability of data and materials}

The data of province-level CO2 emissions are obtained from China Emission Accounts and Datasets. http://www.ceads.net/.And other data are collected from National Statistics Bureau.

\section{http://www.statas.gov.cn/.}

\section{Consent for publication}

\author{
Not applicable.
}

\section{Reference}

Abdeen Mustafa Omer. Focus on low carbon technologies: The positive solution. 2007, 12(9):2331-2357.

Adnan Kasman, Yavuz Selman Duman.CO2 emissions, economic growth, energy consumption, trade and urbanization in new EU member and candidate countries: A panel data analysis.

Arouri M E H, Youssef A B, M'henni H, Rault, C. Energy consumption, economic growth and 
610 Azomahou T, Van Phu N. Economic growth and CO2 emissions: A nonparametric approach.

611 Center for Operations Research and Econometrics (CORE), Université catholique de Louvain,

612 Brussels, Belgium, 2001: 1-28.

613 Bhattacharya M, Paramati S R, Ozturk I, et al. The effect of renewable energy consumption on

614 economic growth: Evidence from top 38 countries. Applied Energy, 2016, 162: 733-741.

615 Breitung J. The local power of some unit root tests for panel data. Nonstationary panels, panel

616 cointegration, and dynamic panels. Emerald Group Publishing Limited, 2001: 161-177.

617 De Hoyos, Rafael E., and Vasilis Sarafidis. Testing for cross-sectional dependence in panel-data

618 models. Stata Journal 2006, 6(4): 1-13.

619 Dong Mei, Xu Zhangyong, Li Cunfang. Simulation of carbon intensity constraints: macroscopic

620 effects, emission reduction effects and structural effects . Management Review, 2019, 31 (05):

$62153-65$.

622 Faisal Faisal,Ruqiya Pervaiz,Nesrin Ozatac,Turgut Tursoy. Exploring the relationship between

623 carbon dioxide emissions, urbanisation and financial deepening for Turkey using the

624 symmetric and asymmetric causality approaches[J]. Environment, Development and

625 Sustainability,2021(prepublish).

626 Fan Zhang, Xiangzheng Deng, Fred Phillips, Chuanglin Fang, Chao Wang. Impacts of

627 industrial structure and technical progress on carbon emission intensity: Evidence from 281

628 cities in China[J]. Technological Forecasting \&amp; Social Change,2020,154.

629 Fei, Li, et al. Energy consumption-economic growth relationship and carbon dioxide emissions 
631 Feng Shuqi, Zhang Jianping, Wang Yaoyao. Analysis of factors influencing Chinese residents'

632 direct living energy consumption carbon intensity .Environmental Engineering, 2018,36 (10):

$633 \quad 184-188$.

634 Galeotti M, Lanza A. Richer and cleaner? A study on carbon dioxide emissions in developing countrie. Energy Policy, 1999, 27(10):565-573.

Gao Lu. Decomposition analysis of influencing factors of changes in carbon emissions in Sichuan Province. Environment and Life, 2014 (16): 7-9.. Agreement[R]. NBER. 1991, working paper 3914

640 Levin A, Lin C F, Chu C S J. Unit root tests in panel data: asymptotic and finite-sample 641 properties. Journal of Econometrics, 2002, 108(1):1-24.

642 Liu Xianzhao, Gao Changchun, Zhang Yong, Zhang Dongshui, Xie Jinning, Song Yan, Wang 643 Zhiqiang. Spatial Heterogeneity of Spatial Dependence Pattern and Influencing Factors of 644 Provincial Carbon Intensity in China .Geography, 2018,38 (05) : 681-690.

645 Liu Yunbo, Research on the influencing factors of carbon intensity in China's power industry. $646 \quad$ Harbin Institute of Technology, 2013.

647 Lotfalipour M R, Falahi M A, Ashena M. Economic growth, CO2 emissions, and fossil fuels 648 consumption in Iran. Energy, 2010, 35(12): 5115-5120.

$649 \mathrm{Lu} \mathrm{H}$. Analysis of the relationship between China's environmental problems and economic 650 development -- taking air pollution as an example. 2000(5):53-59. 
652 Role of renewable and nonrenewable energy[J]. Renewable Energy,2021,172.

653 Maddala G S, Wu S. A comparative study of unit root tests with panel data and a new simple

654 test. Oxford Bulletin of Economics and statistics, 1999, 61(S1): 631-652.

655 Pesaran M H. A simple panel unit root test in the presence of cross-section dependence. Journal

656 of Applied Econometrics, 2007, 22(2): 265-312.

657 Pesaran, M H. General Diagnostic Tests for Cross Section Dependence in Panels. Available at

658 https://papers.ssrn.com/sol3/papers.cfm?abstract_id=572504, (accessed on 4 August, 2004).

659 Roberts J T, Grimes P E. Carbon intensity and economic development 1962-1991: A brief

660 exploration of the environmental Kuznets curve. World Development, 1997, 25(2): 191-198.

661 Soumyananda Dinda. Environmental Kuznets Curve Hypothesis: A Survey[J]. Ecological

662 Economics,2004,49(4).

663 Stan. The characteristics of the new round of energy revolution and the institutional

664 mechanism construction of energy transformation [J]. Financial think tank, 2018(04):17-25 139-

665140.

666 Wang Shaohua, Zhang Wei. Analysis of the carbon intensity factors of Beijing-Tianjin-Hebei

667 based on the whole-region-industry decomposition. Soft Science, 2017, 31 (12): 96-100.

668 Wang Shaojian, Huang Yongyuan. Spatial spillover effect and driving factors of carbon

669 emission intensity of Chinese cities . Acta Geographica Sinica, 2019, 74 (06): 1131-1148.

670 Wang Zhijian,Ben Jebli Mehdi,Madaleno Mara,Doğan Buhari,Shahzad Umer. Does export

671 product quality and renewable energy induce carbon dioxide emissions: Evidence from leading 
672 complex and renewable energy economies[J]. Renewable Energy,2021,171.

673 Zhang, Chuanguo, and Yan Lin. Panel estimation for urbanization, energy consumption and

674 CO2 emissions: A regional analysis in China. Energy Policy, 2012, 49: 488-498.

675 Zhu Ling, Zhang Zhen. Analysis of Influencing Factors of Carbon Emission Intensity in 676 Shanghai. Environmental Science Research, 2011, 24 (1): $20 \sim 26$.

677 Ziqing, Zhu,Yang, Liu,Xin Tian, Yafei Wang, Yan Zhang. CO2 emissions from the 678 industrialization and urbanization processes in the manufacturing center Tianjin in China. 679 Journal of Cleaner Production, 2017(8): 67-75. 